\title{
Using a Fuzzy Light Sensor to Improve the Efficiency of Solar Panels
}

\author{
Suryono Suryono ${ }^{1,2,{ }^{*}}$, Jatmiko Endro Suseno ${ }^{1,2}$, Ainie Khuriati Riza Sulistiati ${ }^{2}$, Tahan Prahara ${ }^{3}$ \\ ${ }^{1}$ Magister of Information System, School of Postgraduate Study, Diponegoro University, Semarang - Indonesia \\ ${ }^{2}$ Department of Physics, Faculty Science and Mathematics, Diponegoro University, Semarang - Indonesia \\ ${ }^{3}$ Department of Informatics Education, Veteran University, Semarang - Indonesia
}

\begin{abstract}
Solar panel efficiency can be increased by improving the quality of photovoltaic material, the effectiveness of electronic circuit, and the light source tracking model. This research is aimed at improving the quality of solar panels by tracking light source using a fuzzy logic sensor. A fuzzy light sensor property is obtained from two LDR (light dependent res istor) light sensors installed in parallel to each other and is given a light separator in between them. Both sensors are mounted on a solar panel. Sensor output is acquired using a 12 bit ADC from an ATSAM3XE microcontroller and is then sent to a computer using WIFI radio. A PID (Proportional-Integral-Derivative) control algorithm is used to manage the position of the solar panel in line with the input given by the fuzzy light sensor. This control mechanism works based on the margin of fuzzy membership from both sensors that is used to move a motor DC that in turn moves the solar panel. Experimental results show a characteristically symmetrical fuzzy membership of both sensors with a reflected correlation of $\mathrm{R}=0.9981$ after gains from both sensors are arranged with a program. Upon being tested in the field, this system was capable of improving the performance of solar panels in gaining power compared to their original fixed position. The discrepancy was evident when the angle of incoming sunlight approached both $0^{\circ}$ and $180^{\circ}$. Further calculations of data acquired by the fuzzy light sensor show increased solar panel power efficiency by up to $5.6 \%$.
\end{abstract}

\section{Introduction}

Energy crisis is one of the biggest problems is most countries now. This issue has great effects on humanity. The underlying reason for this is dependency on fossil fuels with its dwindling available resources. Attempts have been done to generate electricity from other natural resources of energy such as the wind, geothermal, ocean wave, water, and sunlight. It is expected that in 2050 all countries in the world will have used $100 \%$ renewable energy [1].

Solar energy is a form of renewable energy available in its abundance. In tropical countries, solar energy can be harvested all year long. The technology required to convert solar energy into electric power is the photovoltaic solar panel (solar cell) made of semiconductor materials. Solar cell is advantageous from both environmental and efficiency point of views in transmitting power as the electric source can be placed near the facilities using it [2]. The efficiency in converting solar energy into electric power in solar panels is still a hot issue today. Efforts have been carried out to improve this efficiency by among others improving the photovoltaic material, using converter circuits, and tracking sunlight to maximize the power gained by a solar cell.
Monitoring the performance of solar cells connected to one another can improve their efficiency. This monitoring is carried out by mounting a sensor on each solar cell and subsequently reporting their individual conditions. These monitoring results are then sent to a monitoring station via a wireless network [3]. The use of artificial tracking algorithm has been widely used and has been proven to improve the efficiency of solar panels. The use of fuzzy logic algorithm to control solar panels in tracking sunlight has been simulated and has shown its effectiveness in improving the efficiency of solar panels it controlling [4].

Light sensors serve as information input in an artificial intelligent system to control light. Passive Infrared Sensors (PIRs) can be utilized to control lights on the streets as to lower their electric energy consumption [5]. Light sensors can also be used to control the brightness of LCD monitors in order to improve the efficiency of electric power consumption and add to the comfort of the eyes. These two systems can smartly combine ambient light intensity and set point values in order to control brightness, from the lowest to the highest levels [6].

Using a light sensor to know exact positions of a light source (sunlight) can significantly improve the light intensity taken by solar panels. A sensor's ability to track 
sunlight has been proven to improve efficiency in energy management. The sun changes its orientation over time. Therefore, a light sensor must adjust its position to the direction of incoming sunlight. A light sensor should be capable of directing itself to the position of apparent movement of a light source (the sun) on a daily to a monthly basis as to ensure maximum energy gained by solar panels. This means that angular inclination should be perpendicular to the light source as to ensure maximum light intensity exposed to the solar panel [7]. Once this sensor is connected to an automatic solar panel movement system, the performance of the later can improve by up to $25-30 \%$ [8].

Improving the efficiency of energy absorption by solar panels requires a supporting decision system that controls movements of the light sensor. The Opaque Tube technique was developed for sunlight tracking. This system consists of four photocells. It has been proven to improve tracking accuracy by high resolution [9]. The drawback of this technique is during its initial deployment whenever the incoming sunlight does not fall exactly on the sensor's position. This means that when the sunlight only hit the tube, instead of the sensor, it is not detected by the system. Therefore, there is a room for improvement on its construction. The addition of a computer algorithm is expected to minimize this problem and hence, improve its efficiency and effectiveness. This research focuses on the implementation of fuzzy characteristics of two sensors mounted in parallel with a light separator in between them. Output from this fuzzy sensor serves as input for the microcontroller that in turn moves the motor attached on the solar panel.

\section{Research Method}

The system built in this research consists of two sensors, a data acquisition system, and a computer program of proportional-integral-derivative control to move the solar panel. A solar panel coefficient largely depends on the incident angle of sunlight, despite the fact that other factors such as dust, snow, and some other external factors also have their roles. Maximum effectiveness is obtained when the incident angle of the sunlight has an angular coefficientt of $\eta_{\theta}=\sin 90^{\circ}$. The correlation of light intensity and incident angle is shown in Figure 1.

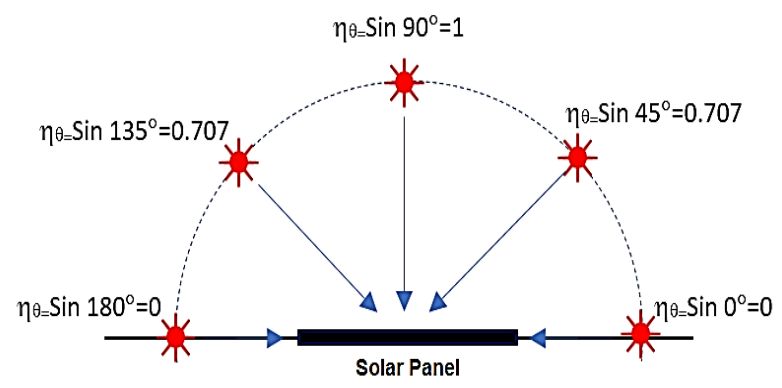

Fig. 1. Light intensity against incident angle of sunlight
This research makes use of a Light Dependent Resistor (LDR), an electronic component of changing resistance value upon contact with light. An LDR's maximum electric resistance is around $10 \mathrm{M} \Omega$ in the dark, whereas its minimum is less than $1 \mathrm{~K} \Omega$ under bright light. The characteristics of an LDR light sensor are given in Figure 2a. In this system, sensors are configured as a voltage divider. In order to produce output voltage equal to that of light intensity, an LDR sensor must be connected in a series with a $1 \mathrm{~K} \Omega$ resistor and given a voltage of 3.3 Volt. Zener diodes are installed at the sensor output to anticipate voltage hikes, so as to protect the other circuit connected to it. A circuit of LDR sensor is depicted in Figure $2 b$.

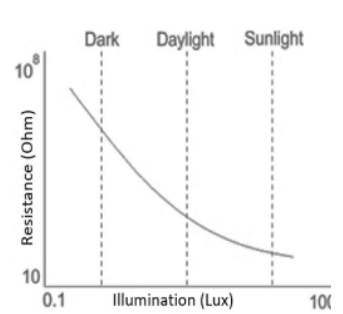

(a)

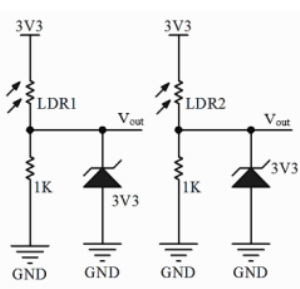

(b)
Fig 2. (a) Characteristics of LDR sensor, and (b) an LDR sensor circuit

The LDR sensor configuration yields maximum values when it is perpendicular to the incoming sunlight, and these values gradually reduce as the sensor is tilted. These values are used to generate fussy data. Sensor placement at certain angles represents weight against angle. A configuration of fuzzy light sensor is shown in Figure $3 \mathrm{a}$, while its side view is given in Figure $3 \mathrm{~b}$. These sensors generate input fuzzy compilation data in the form of angle readings to be processed to obtain faster and more précised data. An ATSAM3X8E is used to acquire fuzzy sensor output and send it to a remote computer.
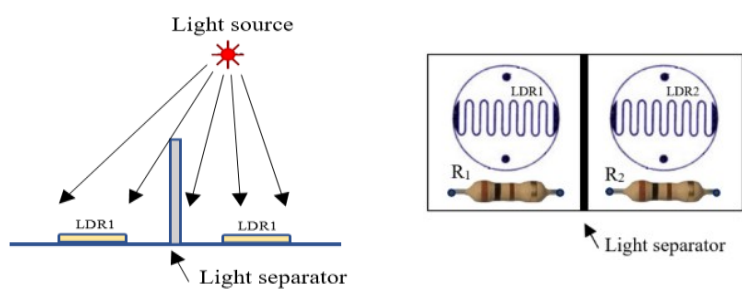

Fig. 3. (a) Configuration of a fuzzy light sensor, and (b) side view of the fuzzy light sensor

They fuzzy light sensor was applied to a 100 watt solar panel. A 32 bit ATSAM3X8E microcontroller was used to acquire data and communicate those to a control computer via the TCIP/IP protocol. A circuit of that data acquisition and communication system is given in Figure 
4. A WIFI radio was utilized as a data communication medium that connects ATSAM3X8E microcontroller to the computer's database system. Fuzzy light sensor output was acquired by a 12 bit $\mathrm{ADC}$ of the ATSAM3X8E microcontroller at channels A0 and A1. These acquired data were then sent to the control computer unit via an Ethernet module set at an IP address of the same class with the WIFI radio and the computer. The computer itself was programmed to using the client-socket and server-socket programming in order to access sensor data in line with the set port and these data were saved in the database every five (5) minutes. Stored data were then processed using the fuzzy logic algorithm according to the characteristics of their membership. Next, a de-fuzzy-fication process was carried out by the computer. This process changes fuzzy values into discreet values. Output values were obtained by adding multiplication results of input compilation membership for each input with the already set output values. The results of these were then divided with the number of multiplication of compilation membership for each input. De-fuzzy-fication transforms fuzzy units into numerical units. These unites were then sent to the microcontroller and set the basis for the Gearbox DC motor movement to position solar panels.

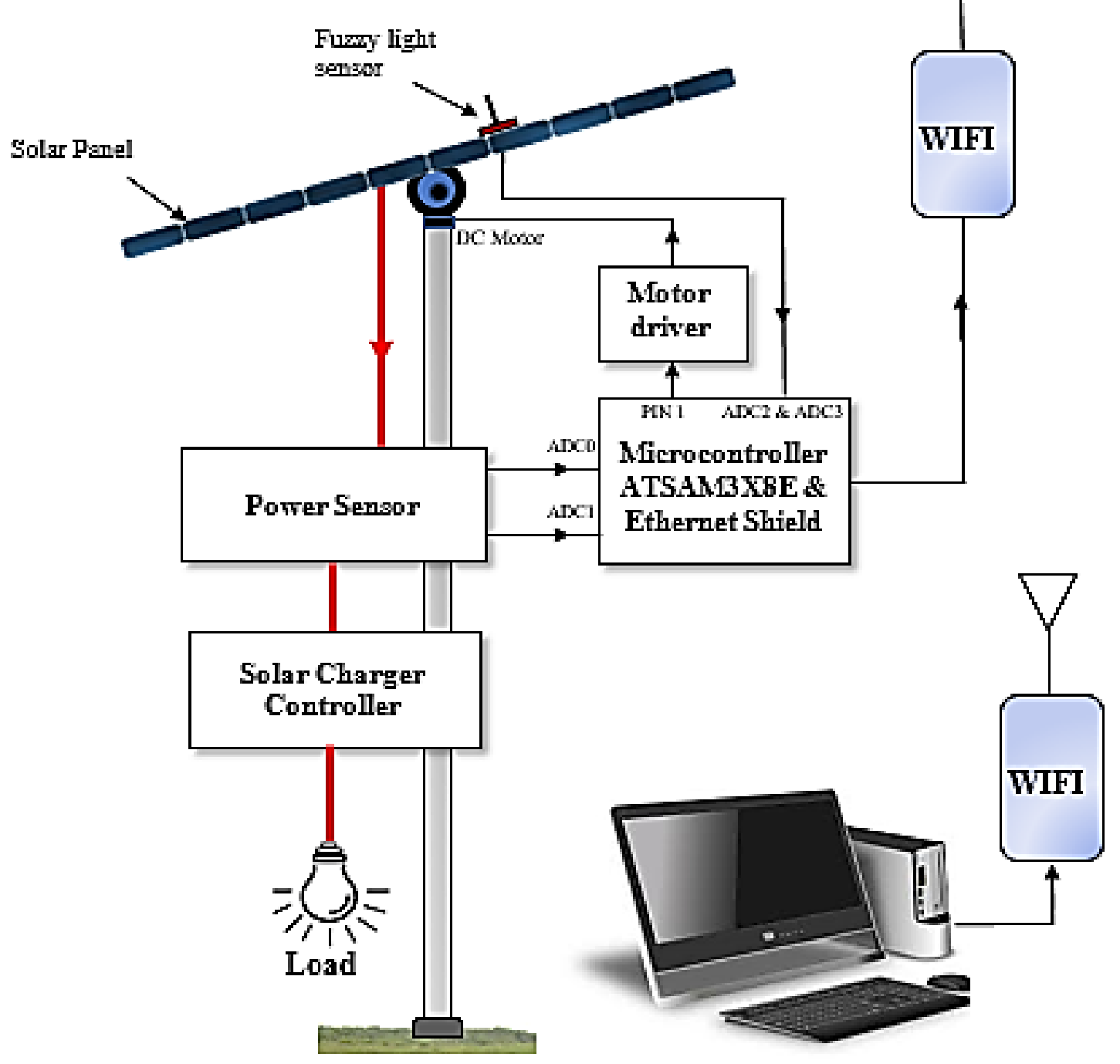

Fig. 4. Instrument system configuration and data acquisition and communication 


\section{Analysis Results}

Characteristics of the fuzzy light sensor were tested by moving a light source above the system. Data acquisition result was recorded by the computer. This result covered angles from $0^{\circ}$ to $180^{\circ}$, adjusted with the position of the sun when the sensor was tested. In this research, the DC motor was set at $1^{\circ}$ accuracy based on the delay time provided by the microcontroller. Results of this characterization are given in Figure 5. Characterization results in Figure 5 show that both sensors have logarithmic characteristics. Linear changes in scanning angles do not affect output voltage linearly but logarithmically instead. It is also shown that both sides of the sensors are not symmetrical. This is due to differences in intrinsic characteristics of the materials used for both sensors. Theoretically, fuzzy membership for both right and left sensors should be symmetrical.

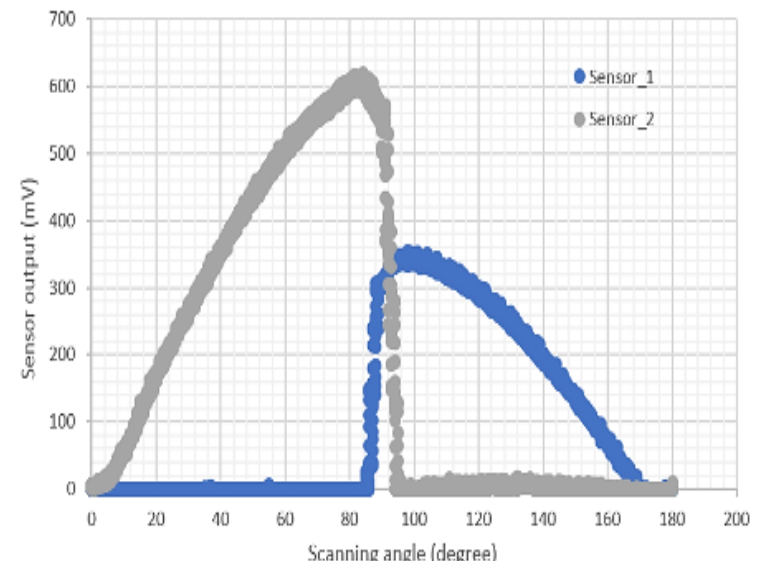

Fig 5. Characterization results for the fuzzy light sensor
Hence, this fuzzy system makes adjustment by carrying out a gain operation for both sensor characteristics with the help of a software. Subsequently, symmetrical values were obtained, with gains of 0.76 on sensor 1 , and 1.34 on sensor 2. Adjustment results for both sensors are given in Figure 6 , the reflected correlation of which is $\mathrm{R}=0.9981$.

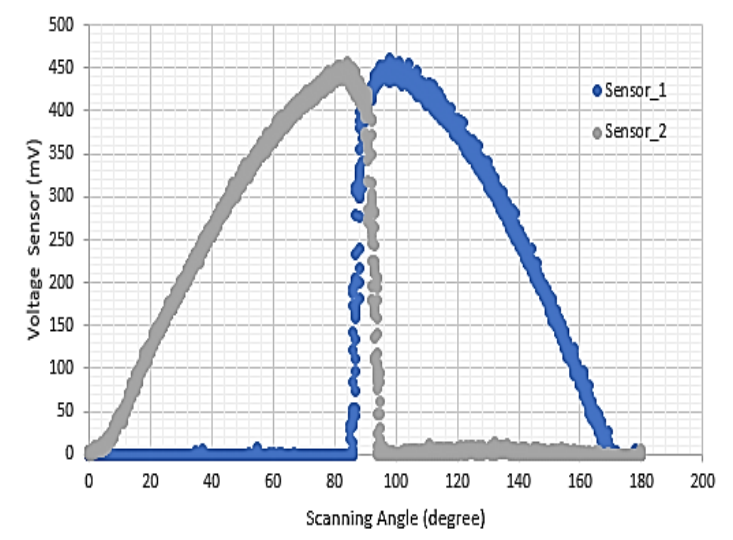

Fig. 6. Results of gain adjustments for both sensors

Fuzzy membership obtained from characterization of both sensors is obtained to control movements of the solar panel. Output from both sensors is used as the basis for solar panel movements. This research made use of the PID (Proportional-IntegralDerivative) control system algorithm for its computer user interface, as shown in Figure 7.

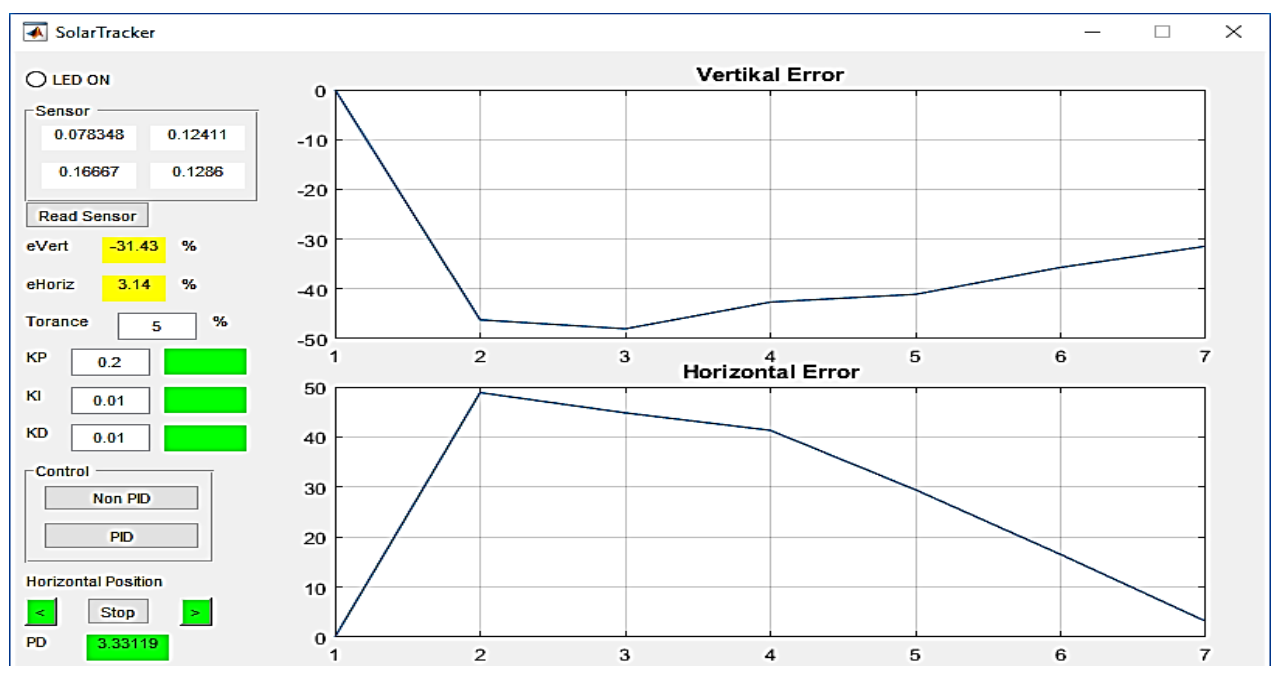

Fig. 7. Computer user interface to show control process for the light fuzzy sensor input in real-time

During each process, differences in output voltage from readings of sensor 1 and sensor 2 are displayed. These differences yield errors that are also displayed in real-time, as shown by the graph above. The less the differences in readings from the two sensors, the more optimum the solar panel position is. The number of iterations toward error $=0$ shows the speed of the control process. Less iterations mean faster control process. 
This control system was then applied in the field. In order to figure out the effect of fuzzy sensor control system, electric power was acquired from two positions. The first was when the solar panel was lying horizontal at $0^{\circ}$ and the second one was during tracking with the fuzzy sensor. Both situations were for the same solar panel and were observed during a slight time-lapse. Readings from both situations are depicted in Figure 8.

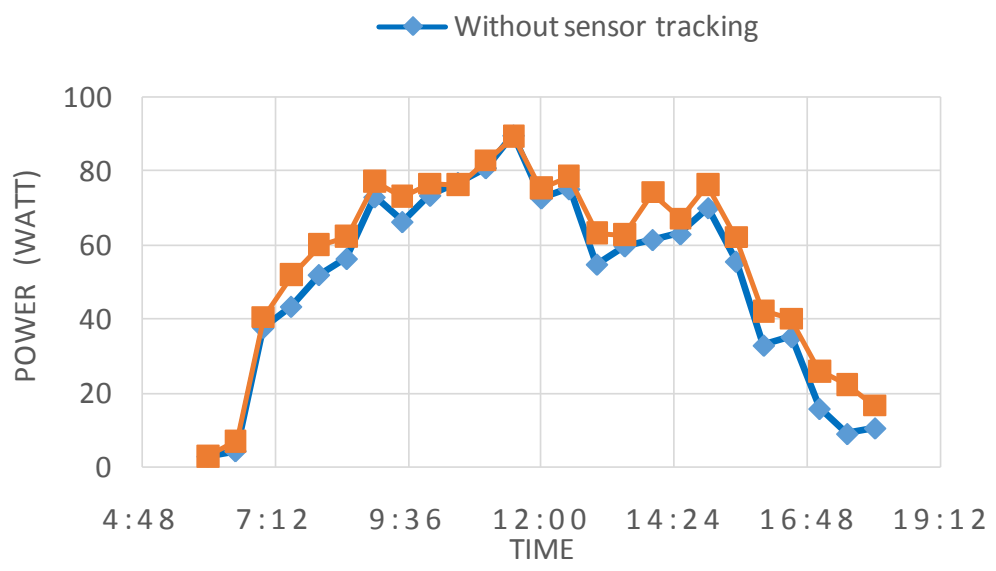

Fig. 8. Results of data acquisition by the solar panel using both fuzzy sensor and fixed position panels

Results of that data acquisition reveal that the power generated by the system employing the fuzzy light sensor is always on top. This means that fuzzy light sensor positively affects solar panel productivity. The effects are evident when the sunlight's angle approaches both $0^{\circ}$ and $180^{\circ}$. This is further supported by the fact that the fixed position solar panel could not get enough sunlight, whereas the fuzzy light sensor solar panel was still capable of positioning itself toward the perpendicular line. Mathematically, it was then calculated that the fuzzy light sensor system improves power gain by up to $5.6 \%$.

\section{Conclusions}

Sensors made of Light Dependent Resistors (LDRs) can be configured to be a light sensor with the properties of a fuzzy logic when they are installed parallel and given a light separator. The characteristics of this LDR fuzzy sensor is exponential with mirroring symmetry of correlation coefficient up to $\mathrm{R}=0.9981$. Fuzzy membership of this sensor output can be utilized as input for the PID (Proportional-Integral-Derivative) control that in turn, is used to control the positioning of a solar panel. Differences in the fuzzy membership of both sensors are the foundation that decides the movement of the DC motor that moves the solar panel.

Fuzzy light sensor as input for PID control is capable for improving power performance of a solar panel compared to its fixed position counterpart. This fact is very evident when the angle of incoming sunlight approaches both $0^{\circ}$ and $180^{\circ}$. Calculation results of data obtained from the fuzzy light sensor show power gaining improvement for solar panels by up to $5.6 \%$.

\section{References}

1. T. N. Ngoc, Q. N. Phung, L. N. Tung, E. R. Sanseverino, P. Romano, F. Viola, Solar Energy, 150 pp. 325-334, (2017).

2. T. Sueyoshi, and D. Wang, Energy Economics, 65 pp. 389-398, (2017).

3. Vivek Kaundal, Amit Kumar Mondal, Paawan Sharma, Kamal Bansal, Engineering Science and Technology, 18 pp. 475-484, (2015).

4. S. Zhang, T. Wang, C. Li, J. Zhang, Y. Wang, "Maximum power point tracking control of solar power generation systems based on type-2 fuzzy logic", 2016 12th World Congress on Intelligent Control and Automation (WCICA), pp. $770-774,(2016)$.

5. Y. M. Jagadeesh, S. Akilesh, S. Karthik, P. Prasanth, Procedia Technology 21 pp. $547-$ 551, (2015).

6. K. Lee, S. Moon, B. Berkeley, S. Kim, Sensors and Actuators A: Physical, 130-131, pp. 214219, (2006).

7. Jeongjae Yoo and Yeonsik Kang, Journal of Automation and Control Engineering Vol. 2, No. 4, December (2014), pp. 417 - 421

8. Stamatescu, I. Făgărășan, G. Stamatescu, N. Arghira, and S. S. Iliescu, Procedia Engineering : 24th DAAAM International Symposium on Intelligent Manufacturing and Automation 69 pp. $500-507,(2014)$.

9. D. Fontani, P. Sansoni, F. Francini, D. Jafrancesco, L. Mercatelli, and E. Sani, International Journal of Photoenergy 2011, (2011), pp. 1-9. 